\title{
Anomaly-free Abelian gauge symmetries with Dirac scotogenic models
}

\author{
Nicolás Bernal॰* \\ Centro de Investigaciones, Universidad Antonio Nariño, Carrera 3 Este No. 47A-15, Bogotá, Colombia \\ Julián Calle $\odot^{\dagger}$ and Diego Restrepo® $\odot^{*}$ \\ Instituto de Física, Universidad de Antioquia, Calle 70 No. 52-21, \\ Apartado Aéreo 1226, Medellín, Colombia
}

(Received 22 February 2021; accepted 10 May 2021; published 27 May 2021)

\begin{abstract}
We perform a systematic analysis of standard model extensions with an additional anomaly-free gauge $U(1)$ symmetry, to generate Dirac neutrino masses at one loop. Under such symmetry, standard model fields could either transform or be invariant, corresponding to an active $U(1)_{X}$ or a dark $U(1)_{D}$ symmetry, respectively. Having an anomaly-free symmetry imposes nontrivial conditions to the number and charges of the unavoidable new states. We perform an intensive scan, looking for nonanomalous solutions for a given number of extra chiral fermions. In particular, we concentrate on solutions giving rise to scotogenic neutrino masses via the effective Dirac mass operator. We study the cases where the Dirac mass operator with dimension 5 or 6 is mediated by Dirac or Majorana states and corresponds to an active $U(1)_{X}$ or a dark $U(1)_{D}$ symmetry. Finally, we comment on the solutions featuring no massless chiral fermions.
\end{abstract}

DOI: $10.1103 /$ PhysRevD.103.095032

\section{INTRODUCTION}

The standard model (SM) of particle physics is a very successful theory, even though it has to be extended in order to account for neutrino masses and dark matter (DM). The interpretation of neutrino experimental data in terms of neutrino oscillations is compatible with both Majorana and Dirac neutrino masses [1], with no theoretical preference for either of the possibilities. However, most of the proposals in the literature assume that neutrinos are Majorana in nature (see Ref. [2] for a review), whereas mass generation mechanisms for Dirac type neutrinos are less studied but have recently received increased attention (see, i.e., Refs. [3-43]).

To explain Dirac neutrino masses, right-handed neutrinos (RHNs) have to be introduced; however, that is not enough. An extra local symmetry is also required to guarantee proper total lepton number conservation [7]. Even so, the resulting Yukawa couplings can turn out to be too small, of the order $\mathcal{O}\left(10^{-10}\right)$ or even smaller, if Dirac neutrino masses are induced directly from the SM Higgs mechanism $[25,44]$. Nevertheless, if the symmetry forbids

\footnotetext{
*nicolas.bernal@uan.edu.co

†julian.callem@udea.edu.co

"restrepo@udea.edu.co
}

Published by the American Physical Society under the terms of the Creative Commons Attribution 4.0 International license. Further distribution of this work must maintain attribution to the author(s) and the published article's title, journal citation, and DOI. Funded by SCOAP ${ }^{3}$. the tree-level contribution driven by the SM Higgs, a Diracseesaw mechanism can be implemented. For example, the type-I Dirac seesaw could appear in the context of anomaly-free gauge $U(1)_{B-L}$ symmetries [7]. At one loop, the heavy particles in the radiative seesaw can be fully associated with an Abelian gauge dark symmetry $U(1)_{D}$ with the lightest of them as a DM candidate [32,45-47], as well as to an active Abelian gauge symmetry $U(1)_{X}$, like a $U(1)_{B-L}[24,25,32]$. Until now, the studies of one-loop Dirac neutrino masses have typically focused on finding specific anomaly-free solutions of these two kinds of symmetries (see, e.g., Refs. [30,31,33,42,48,49]). Here, we present a complete set of relevant anomaly-free solutions to the general problem of the generation of Dirac neutrino masses at one loop with chiral singlet fermions. Each of the solutions leads to a unique model with its specific phenomenological implications. Our method can be easily applied to find the full set of anomaly-free solutions to a well-defined phenomenological problem.

In this work, we look for anomaly-free solutions to SM extensions with an additional $U(1)$ gauge symmetry, giving rise to scotogenic Dirac neutrino masses. For that purpose, in Sec. II we study the conditions to have a nonanomalous $U(1)$ gauge symmetry. In particular, we show that the case where the SM is extended with an active Abelian gauge symmetry $U(1)_{X}$ with nonvanishing generation independent charges and a set of $N^{\prime}$ singlet chiral fermions shares the same anomaly-free solutions as an Abelian gauge dark symmetry $U(1)_{D}$ with $N^{\prime}+3$ singlet chiral fermions having at least three equal charges. In Sec. III, we focus 
on solutions giving rise to scotogenic neutrino masses via the effective Dirac mass operator. For that purpose, we perform an intensive scan, looking for nonanomalous charge assignments for a given number of extra chiral fermions. We study the cases where the Dirac mass operator, with dimension 5 or 6 , is mediated by Dirac or Majorana states and corresponds to an active $U(1)_{X}$ or a dark $U(1)_{D}$ symmetry. We pay special attention to the solutions where all chiral fermions obtain mass via the spontaneous symmetry breaking (SSB) of the $U(1)$. Finally, in Sec. IV our conclusions are presented.

\section{ANOMALY CONDITIONS}

We consider an extension of the SM with an additional $U(1)_{X}$ gauge symmetry and $N^{\prime}$ right-handed chiral fields $\psi_{\rho}$ singlets under the $\mathrm{SM} S U(3)_{c} \otimes S U(2)_{L} \otimes U(1)_{Y}$ group, with charges $n_{\rho}$ under the $U(1)_{X}$, where $\rho=1, \ldots, N^{\prime}$. Additionally, we assume that the SM right-handed chiral fermions transform under the $U(1)_{X}$, with charges denoted with the same name of the field. ${ }^{1}$ To avoid having an anomalous $U(1)_{X}$, the three linear anomaly conditions are

$$
\begin{aligned}
{\left[S U(3)_{C}\right]^{2} U(1)_{X}:[3 u+3 d]+[3 \times 2 Q]=0, } & \\
{\left[S U(2)_{L}\right]^{2} U(1)_{X}: } & {[2 L+3 \times 2 Q]=0, } \\
{\left[U(1)_{Y}\right]^{2} U(1)_{X}: } & {\left[(-2)^{2} e+3\left(\frac{4}{3}\right)^{2} u+3\left(-\frac{2}{3}\right)^{2} d\right] } \\
+ & {\left[2(-1)^{2} L+3 \times 2\left(\frac{1}{3}\right)^{2} Q\right]=0 . }
\end{aligned}
$$

As they only depend on the SM fermions, three of their $X$ charges can be expressed in terms of the other two [32,50-52], chosen to be $e$ and $L$, as

$$
u=-e-\frac{2}{3} L, \quad d=e+\frac{4}{3} L, \quad Q=-\frac{1}{3} L .
$$

We note that the quadratic anomaly condition in $U(1)_{X}$ is trivially satisfied. However, the mixed gauge-gravitational $[\mathrm{Grav}]^{2} U(1)_{X}$ and the cubic $\left[U(1)_{X}\right]^{3}$ Anomalies do depend on the extra fermion charges $n_{\rho}$, and therefore two additional conditions have to be imposed in order to avoid an anomalous $U(1)_{X}[32]$,

$$
\sum_{\rho=1}^{N^{\prime}} n_{\rho}+3 m=0, \quad \sum_{\rho=1}^{N^{\prime}} n_{\rho}^{3}+3 m^{3}=0
$$

where $m \equiv e+2 L$. Equation (4) can be rewritten as

\footnotetext{
${ }^{1} Q$ and $L$ are the $X$ charges of the fermion doublets $Q^{\dagger}$ and $L^{\dagger}$, respectively.
}

$u=\frac{4 L}{3}-m, \quad d=m-\frac{2 L}{3}, \quad Q=-\frac{L}{3}, \quad e=m-2 L$.

Finally, we note that the SM Higgs must have a $X$ charge

$$
h=-e-L=L-m,
$$

to guarantee that SM quarks and charged leptons acquire masses through the standard Higgs mechanism. ${ }^{2}$ Along these lines, we also assume that the singlet chiral fermions $\psi_{\rho}$ only acquire mass through the SSB of the extra $U(1)_{X}$ symmetry. This excludes solutions with vectorlike states. We note that the existence of fields charged under both hypercharge and $U(1)_{X}$ induce at loop level the kinetic mixing operator $\mathcal{L} \supset \frac{\epsilon}{2} B^{\mu \nu} X_{\mu \nu}$, where $B^{\mu \nu}$ and $X^{\mu \nu}$ are the field strengths related to the $U(1)_{Y}$ and the extra $U(1)_{X}$, respectively. The dimensionless parameter $\epsilon$ depends on the masses of the particles in the loop, as well as their specific charge assignment and the gauge couplings under the two $U(1)$ symmetries [53-55].

It is interesting to note that the conditions in Eq. (5) are completely equivalent to the ones coming from a scenario where the SM is extended with a dark $U(1)_{D}$ gauge symmetry with $N=N^{\prime}+3$ right-handed singlet chiral fermions, $N^{\prime}$ of them with the charges $n_{\rho}$ and three with charge $m$, and where the SM is invariant (hence a dark symmetry). Even if comparable, there is a major technical advantage of the latter approach: if the SM is extended with an additional dark $U(1)_{D}$ gauge symmetry (under which it is uncharged) and $N$ right-handed chiral fields singlets under the SM group, the $U(1)_{D}$ is not anomalous if the Diophantine equations

$$
\sum_{\rho=1}^{N} n_{\rho}=0 \quad \text { and } \quad \sum_{\rho=1}^{N} n_{\rho}^{3}=0
$$

coming from the mixed gauge-gravitational $[\mathrm{Grav}]^{2} U(1)_{D}$ and cubic $\left[U(1)_{D}\right]^{3}$ conditions are fulfilled. It is well-known that the solution of Eq. (8) is highly nontrivial [4,8,56-59]. However, for a $U(1)$ symmetry, it can be parametrized as a function of two sets of integers $\ell$ and $k$, with dimensions $(N-3) / 2$ and $(N-1) / 2$ for $N$ odd or $N / 2-1$ and $N / 2-1$ for $N$ even [58]. We have implemented an official PYTHON package called Anomalies ${ }^{3}$ to obtain the solution associated with any set of integers $\ell$ and $k$.

In general, a very large number of solutions can be found for a given $N$. For example, for $N \leq 9, \mathcal{O}\left(10^{9}\right)$ different combinations of $\ell$ and $k$ exist, if one allows charges to reach a maximum absolute value of 30 . However, the number of chiral solutions (i.e., without

\footnotetext{
${ }^{2}$ In particular, if $h=0$ a gauge symmetry with SM-fermion charge $X=m(B-L)$ is obtained, where $B-L$ are the baryonminus-lepton charges.

${ }^{3}$ See Ref. [60].
} 
featuring vectorlike states) reduces to around 30000 . We note that nearly half of them have at least a pair of repeated charges, corresponding to a requirement for having at least a couple of RHNs. This large number of solutions is too large in practice to perform a phenomenological study. However, a manageable set of solutions can be found by imposing further constraints. For example, one can look for realizations of the effective Dirac mass operator of dimensions 5 and 6 through scotogenic models mediated by heavy chiral fermions. This possibility will be explored in the next section.

\section{DIRAC SCOTOGENIC MODELS}

In this section, we look for anomaly-free $U(1)_{D}$ or $U(1)_{X}$ gauge extensions of the $\mathrm{SM}$, with $N$ or $N-3$ singlet chiral fermions, respectively, realizing one-loop effective Dirac neutrino mass operators [61,62]. In the two-component spinor notation, they can be written as

$$
\begin{gathered}
\mathcal{L}_{\text {eff }}=h_{\nu}^{\alpha i}\left(\nu_{R \alpha}\right)^{\dagger} \epsilon_{a b} L_{i}^{a} H^{b}\left(\frac{S^{*}}{\Lambda}\right)^{\delta}+\text { H.c. } \\
\text { with } \quad i=1,2,3
\end{gathered}
$$

and $\delta=1$ or 2 for dimension 5 (D-5) or 6 (D-6) operators, respectively. Here $h_{\nu}^{\alpha i}$ correspond to dimensionless induced couplings, $\nu_{R \alpha}$ are at least two RHNs $(\alpha=1,2, \ldots)$ with the same $D$ or $X$ charge $\nu, L_{i}$ are the lepton doublets with $X$ charge $-L, H$ is the SM Higgs doublet with $X$ charge $h=L-m, S$ is the complex singlet scalar responsible for the SSB of the anomaly-free gauge symmetry with $D$ or $X$ charge $s=-\nu / \delta$ or $s=-(\nu+m) / \delta$, respectively, and $\Lambda$ is a scale of new physics, which is parametrically the typical mass scale of the new (heavy) states. In general, after the $\mathrm{SSB}$, a remnant $\mathbb{Z}_{|s|}$ discrete symmetry is left, which can guarantee the stability of a potential DM candidate [46,63]. Additionally, we note that the SM Higgs boson can mix with the scalar $S$, after symmetry breaking. In general, the mixing between these two bosons can lead to a stabilization of the metastable electroweak vacuum of the SM [64].

The left (right) panels of Fig. 1 present the topologies realizing the D-5 (D-6) effective Lagrangian in Eq. (9), if one only allows SSB masses for the singlet chiral fermions. The different diagrams are labeled following the notation used in Ref. [21]. Here $X_{1}$ and $X_{2}$ correspond to the singlet chiral fermion fields. Without loss of generality, one can choose $X_{1}$ to be a right-handed field $\chi_{R}$ with $D$ or $X$ charge $r$. After the SSB of the extra Abelian symmetry, a heavy Majorana or Dirac mediator is generated depending if one chooses $X_{2}$ to be the same $\chi_{R}$ or a new $\left(\chi_{L}\right)^{\dagger}$, with $D$ or $X$ charge $l$. In the last two rows, $X_{3}$ is a doublet vectorlike fermion with an additional coupling to the Higgs and a singlet chiral fermion. Finally, $Y_{i}$ corresponds to either singlet or doublet inert scalars according to the specific vertex. For example, for the topology T1-3-E, $Y_{1}$ is an inert doublet scalar, while $Y_{2}$ is an inert singlet scalar.

The realizations of the D-5 operator featuring heavy Dirac (Majorana) mediators are shown in the left (right) panel of Fig. 2. The realizations of the D-6 operators are almost identical, but with an additional extra external singlet scalar line and are therefore not shown. For example, the upper left diagram in Fig. 2, labeled as T13 -E-D, is realized for a dark $U(1)_{D}$ symmetry if the flux of the $D$ charges in each vertex satisfy

$\eta=r, \quad s=r+l, \quad \sigma=-l-\nu, \quad \sigma=\eta$,

and therefore, the fermion chiral $D$ charges obey

$$
\nu+l+r=0 .
$$

The scalar $D$ charges can be expressed as a function of $l$ and $r$ as

$$
s=-\nu=r+l, \quad \sigma=\eta=r .
$$

In general, for realizing one-loop Dirac neutrino masses, one requires

$$
\nu+\delta(l+r)+m=0,
$$

with $m=0$ for a gauge $U(1)_{D}$ symmetry, and $l=r$ for realizations with massive Majorana mediators.

As mentioned previously, in order to limit the total number of solutions that cancel the anomaly induced by the additional $U(1)_{X}$ or $U(1)_{D}$ symmetries, the following restrictions are taken into account:

(1) By construction, all new chiral fermions have to be charged under $U(1)_{X}$ or $U(1)_{D}$ symmetries, i.e., solutions with vanishing charges are disregarded.

(2) For the chiral fields, the maximal charge allowed (in absolute value) is 30 .

(3) Solutions with vectorlike fermions are disregarded, i.e., the ones containing two opposite charges.

(4) At least two charges have to be equal. Their corresponding fields are identified with the RHNs.

(5) In the case of a $U(1)_{X}$ symmetry, another set of three equal charges is required.

(6) We restrict ourselves to $N \leq 9$ fields, with charges satisfying the two Diophantine conditions in Eq. (8), and take the minimal charge (in absolute value) to be positive. We note that there are no solutions for $N \leq 5$ with at least two equal charges [5,65].

(7) The charge assignment may not allow all chiral fields to acquire masses via the SSB. We only consider solutions that have at most two massless chiral fields.

(8) We want RHN masses to be generated radiatively. That implies that all vertices between $S$, the RHN, 

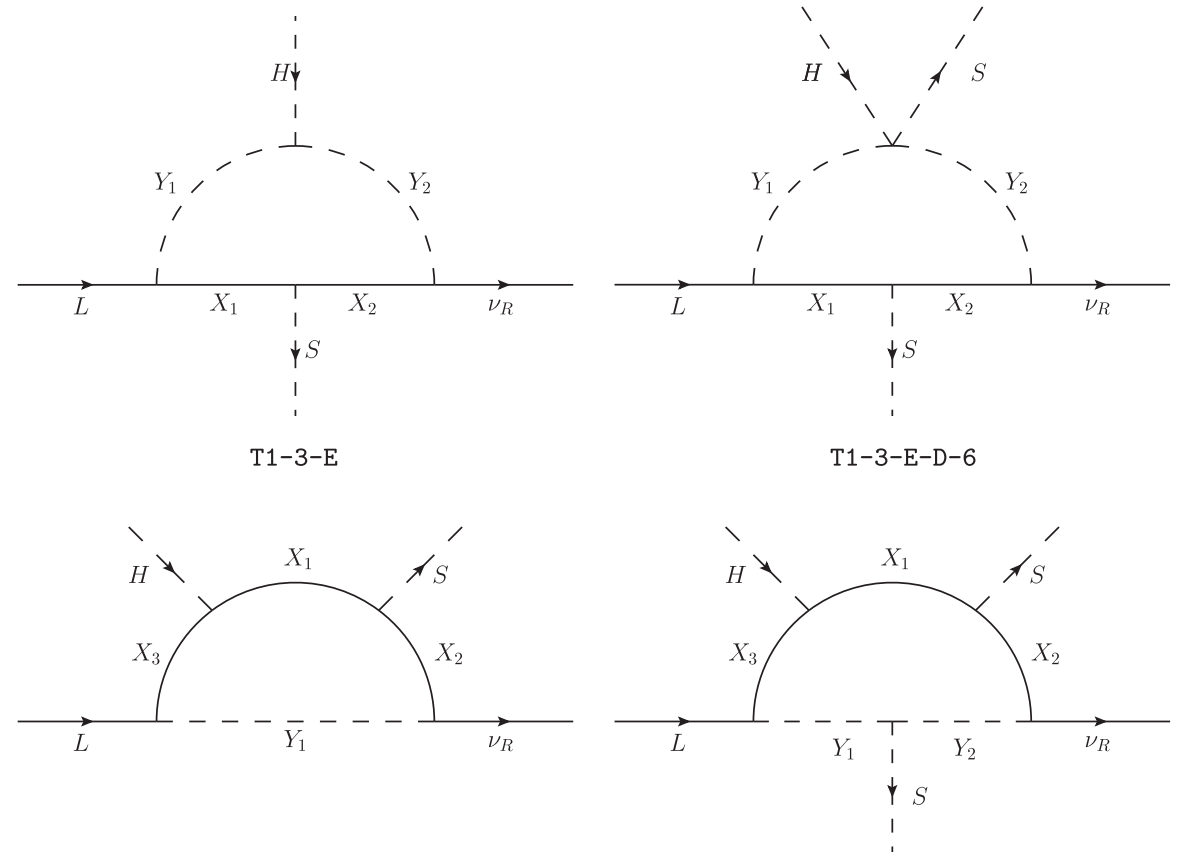

T1-2-A

T1-2-A-D-6
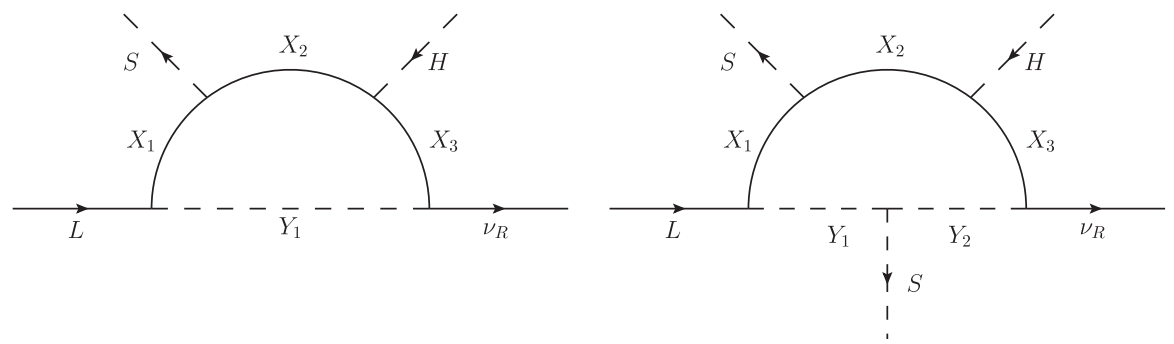

T1-2-B

T1-2-B-D-6

FIG. 1. Topologies leading to one-loop Dirac neutrino masses via the dimension 5 (left) or dimension 6 (right) operators described in Eq. (9). Here we follow the notation used in Ref. [21].

and the other chiral fields should be forbidden by the symmetries.

(9) A DM candidate should flow in the loop, in order to have a scotogenic solution.

Additionally, we note that, for a fixed number of chiral fields and a given topology, different solutions could share the same qualitative behavior. For example, there are four solutions for a $U(1)_{D}$ symmetry with Dirac mediators, $N=6$ chiral fields, and neutrino masses generated via the D-5 operator: $(1,-2,-3,5,5,-6),(2,-3,-10,13,13,-15)$, $(3,-4,-21,25,25,-28)$, and $(3,-5,-12,17,17,-20)$. However, they all feature the same phenomenology, i.e., two RHNs and four other massive chiral fermions. Therefore, in this case only one solution (the one with the smallest charge in absolute value) will be reported in the following.

The solutions of the Diophantine equations satisfying all the previously enumerated conditions are shown in Tables I and II for the D-5 and D-6 operators, respectively. The solutions for $N$ extra chiral fermions are parametrized as a function of two sets of integers $l$ and $k$ (first three columns). The fourth column shows the charge assignments, whereas the fifth shows the general common denominator (GCD) of the original solution. ${ }^{4}$ The last four columns correspond to the charges for the RHNs in the cases of $U(1)_{D}$ symmetry with a Dirac mediator, $U(1)_{D}$ symmetry with a Majorana mediator, $U(1)_{X}$ symmetry with a Dirac mediator, and $U(1)_{X}$ symmetry with a Majorana mediator. ${ }^{5}$ We note that, even if most of the solutions contain at least one massless chiral fermion, there are few solutions without (highlighted in bold): six in the D-5 case and three in the D-6 case. Regarding these solutions without massless fermions, a few comments are in order:

\footnotetext{
${ }^{4}$ The solutions presented were normalized to have a GCD equal to 1 .

${ }^{5} \mathrm{~A}$ small set of the solutions has already been explored in Refs. [4,25,32,42,43,66].
} 

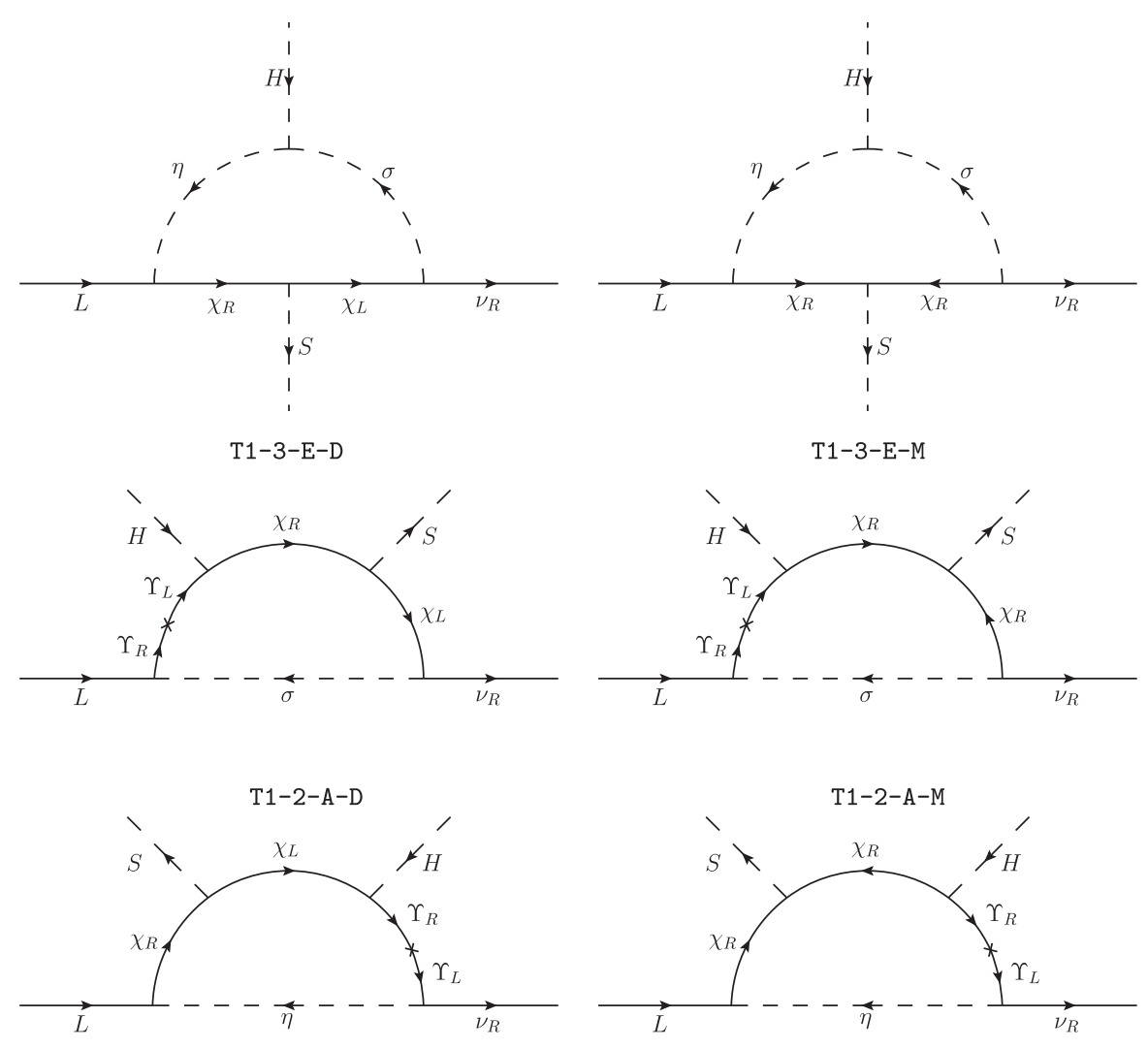

T1-2-B-D

T1-2-B-M

FIG. 2. Diagrams for the D-5 realizations of Dirac neutrinos mass with Dirac heavy mediators (left) and Majorana heavy mediators (right). Similar diagrams, but with an extra external singlet scalar line, are expected for the D-6 realization.

(i) For a $U(1)_{D}$ symmetry, a new solution for the D-5 operator $(\delta=1)$ with a Dirac mediator and a minimal set of $N=6$ fermion chiral fields was found, corresponding to $(1,-2,-3,5,5,-6){ }^{6}$ In this case, there are two RHNs with charges $\nu=5$. The other four chiral fermions combine in pairs $[(1,-6)$ and $(-2,-3)]$ to form a couple of Dirac fermions that obtain mass via the extra scalar $S$ with charge $s=-\nu / \delta=-5$. The two Dirac fermions are stable and give rise to a multicomponent DM scenario. The solution mediated by a Majorana state is not viable because it will require a fermion with a charge $r=-\nu / 2=-5 / 2$, which is absent. Finally, there cannot be solutions for the $U(1)_{X}$ symmetry, as there is no charge repeated three times.

(ii) The solution with $N=9$ fermion chiral fields with charges $(1,1,-4,-5,9,9,9,-10,-10)$ is recovered [42]. It includes three RHNs with charge 9, together with two pairs $(1,-10)$ and one pair $(-4,-5)$. This scenario also features multicomponent DM.

\footnotetext{
${ }^{6}$ This solution was very recently presented in Ref. [43].
}

(iii) In addition, we found another new solution with $N=9:(1,-2,3,4,6,-7,-7,-7,9)$. It includes three RHNs with charge -7 and three massive singlet Dirac fermions $(1,6),(-2,9)$, and $(3,4)$, all of which can be independent DM particles.

(iv) The solution with $N=9$ corresponding to $(1,-2,-2$, $-2,5,-7,8,9,-10)$, contains three RHNs with charge -2 . The condition in Eq. (13) is fulfilled for the Dirac mediator $(-7,9)$ and for the Majorana mediator associated with the chiral fermion of charge 1. Both of them acquire masses from the SSB of the scalar singlet with charge 2. This scalar also generates a mixed sector of three chiral fermions $(5,-7,9)$ and a massive Dirac fermion $(8,-10)$.

(v) The last solution for $N=9$ corresponds to a $U(1)_{X}$ symmetry and a Dirac mediator and has the charge assignment $(1,2,-6,-6,-6,8,9,9,-11)$. It includes two RHNs with charge 9 and four chiral fermions that pair like $(1,2)$ and $(8,-11)$ to get a mass via the scalar $S$ with charge -3 .

(vi) Let us consider now the D-6 operators. For a $U(1)_{X}$ symmetry with Dirac mediators, there is a new solution with $N=7$ corresponding to $(3,3,3$, $-5,-5,-7,8)$. It includes two RHNs with charge 
TABLE I. Set of charges satisfying the Diophantine equations together with the conditions enumerated in the text, for $N$ extra chiral fermions, featuring Dirac neutrino masses generated by D-5 operators. The last four columns correspond to the charges for the RHNs in the cases of $U(1)_{D}$ or $U(1)_{X}$ symmetries and Majorana (Maj.) or Dirac mediators. The solutions without massless chiral fermions are highlighted with a bold font.

\begin{tabular}{|c|c|c|c|c|c|c|c|c|c|}
\hline \multirow[b]{3}{*}{$\mathrm{N}$} & \multirow[b]{3}{*}{$\ell$} & \multirow[b]{3}{*}{$\mathrm{k}$} & \multirow{2}{*}{\multicolumn{3}{|c|}{ Solutions }} & \multicolumn{4}{|c|}{$\nu_{R}$} \\
\hline & & & & & & \multicolumn{2}{|c|}{$U(1)_{D}$} & \multicolumn{2}{|c|}{$U(1)_{X}$} \\
\hline & & & Charges & GCD & Ref. & Dirac & Majorana & Dirac & Majorana \\
\hline 6 & $(-1,1)$ & $(-2,0)$ & $(1,-2,-3,5,5,-6)$ & 1 & [43] & $(5)$ & & & \\
\hline 7 & $(-1,1)$ & $(-1,0,-1)$ & $(1,2,2,-3,-3,-3,4)$ & 1 & {$[43]$} & $(-3)$ & & & \\
\hline 7 & $(1,-1)$ & $(-2,-5,-4)$ & $(1,1,-3,-4,6,6,-7)$ & 1 & & $(1,6)$ & $(6)$ & & \\
\hline 7 & $(-1,1)$ & $(-1,-2,-1)$ & $(1,3,-4,5,-6,-6,7)$ & 1 & & $(-6)$ & $(-6)$ & & \\
\hline 8 & $(-1,-5,-3)$ & $(-6,-4,-7)$ & $(1,1,2,3,-4,-4,-5,6)$ & 1 & & $(-4,1)$ & $(-4)$ & & \\
\hline 8 & $(-1,2,-2)$ & $(-7,4,0)$ & $(1,2,2,2,-3,-5,-6,7)$ & 1 & & (2) & & & \\
\hline 8 & $(1,2,1)$ & $(-5,-10,-11)$ & $(1,2,2,4,-5,-5,-7,8)$ & 1 & & $(-5)$ & & & \\
\hline 8 & $(1,-3,-2)$ & $(-4,-9,-5,-3)$ & $(1,3,3,3,-5,-7,-7,9)$ & 1 & & & & $(-7)$ & \\
\hline 8 & $(2,-1,0)$ & $(-5,-9,-1,0)$ & $(1,2,3,5,-6,-6,-9,10)$ & 1 & & $(-6)$ & $(-6)$ & & \\
\hline 8 & $(-1,0,-1)$ & $(-2,1,-1)$ & $(2,-5,-5,-5,7,8,8,-10)$ & 1 & {$[25]$} & $(8)$ & & $(8)$ & \\
\hline 8 & $(0,-1,0)$ & $(-1,-5,-1,1)$ & $(1,1,1,-5,-7,11,11,-13)$ & 2 & & & & (11) & \\
\hline 8 & $(-4,-1,0)$ & $(-1,0,-8,8)$ & $(3,-4,-5,8,8,-11,-12,13)$ & 1 & & (8) & $(8)$ & & \\
\hline 9 & $(-2,0,2)$ & $(-1,1,0,-1)$ & $(1,1,-4,-5,9,9,9,-10,-10)$ & 1 & [42] & (9) & & & (1) \\
\hline 9 & $(-4,-5,3)$ & $(-2,0,-1,-2)$ & $(3,3,-4,5,5,-6,-8,-8,10)$ & 1 & & $(-8,3,5)$ & & & \\
\hline 9 & $(5,0,1)$ & $(-1,-2,0,2)$ & $(1,1,-5,-7,12,14,14,-15,-15)$ & 3 & & $(-15,1,14)$ & (14) & & \\
\hline 9 & $(1,4,-1)$ & $(-2,-5,-4,8)$ & $(1,1,1,2,5,-6,-6,-6,8)$ & 1 & & $(-6,1)$ & & & \\
\hline 9 & $(-1,0,1)$ & $(-1,1,-2,-1)$ & $(1,-3,-3,-3,-5,8,8,8,-11)$ & 1 & & $(-3,8)$ & & & \\
\hline 9 & $(-7,-5,3)$ & $(-6,-4,-5,2)$ & $(1,-2,-2,-4,7,-9,11,11,-13)$ & 1 & & $(-2,11)$ & $(-2)$ & & \\
\hline 9 & $(3,-2,3)$ & $(-2,-1,-2,4)$ & $(4,4,4,-5,-9,-10,-10,11,11)$ & 1 & & & & $(-10,11)$ & \\
\hline 9 & $(-2,-6,5)$ & $(-5,-1,-3,-6)$ & $(1,1,2,2,3,-5,-6,-6,8)$ & 1 & & $(2)$ & $(-6)$ & & \\
\hline 9 & $(-2,3,2)$ & $(-2,-9,-5,9)$ & $(1,-2,3,4,6,-7,-7,-7,9)$ & 1 & & $(-7)$ & & & \\
\hline 9 & $(-3,-1,5)$ & $(-9,3,-4,-1)$ & $(1,2,-3,4,-5,-6,8,8,-9)$ & 1 & & $(8)$ & & & \\
\hline 9 & $(-8,-7,5)$ & $(-9,3,-4,-2)$ & $(1,-2,-2,-2,5,-7,8,9,-10)$ & 1 & & $(-2)$ & $(-2)$ & & \\
\hline 9 & $(-4,-1,-4)$ & $(-3,-5,1,-4)$ & $(2,-3,4,4,4,-6,-7,-7,9)$ & 1 & & (4) & & $(-7)$ & \\
\hline 9 & $(-3,1,-2)$ & $(-4,-3,-6,-3)$ & $(2,-3,-3,-3,-5,7,7,8,-10)$ & 1 & & $(-3)$ & & & \\
\hline 9 & $(-3,6,5)$ & $(-1,-6,2,-7)$ & $(2,-3,-3,-3,-6,7,7,11,-12)$ & 2 & & & & (7) & \\
\hline 9 & $(-4,2,-3)$ & $(-2,-5,5,-6)$ & $(1,2,-6,-6,-6,8,9,9,-11)$ & 2 & & (9) & & (9) & \\
\hline 9 & $(1,-1,2)$ & $(-2,-1,0,-2)$ & $(2,-3,4,6,6,-7,-10,-11,13)$ & 1 & & (6) & $(6)$ & & \\
\hline 9 & $(-2,-1,-3)$ & $(-1,-4,-3,-4)$ & $(4,4,6,6,-7,-7,-7,-12,13)$ & 1 & & & & (6) & \\
\hline 9 & $(-4,1,2)$ & $(-1,-4,2,1)$ & $(1,-2,-2,-3,-3,-3,14,20,-22)$ & 2 & & & $(-2)$ & & \\
\hline 9 & $(1,4,7)$ & $(-1,6,4,-5)$ & $(1,-2,-2,5,-7,-7,14,18,-20)$ & 4 & & & $(-2)$ & & \\
\hline 9 & $(8,-1,0)$ & $(-1,-6,-3,-6)$ & $(1,9,-12,-21,-21,24,24,24,-28)$ & 18 & & & $(24)$ & $(-21)$ & \\
\hline
\end{tabular}

-5 and an extra scalar with charge 1 that gives mass to an extra singlet Dirac fermion $(-7,8)$, which is also a DM candidate.

(vii) There is another solution with $N=9$ given by $(1,-3$, $8,8,8,-12,-12,-17,19)$. It includes two RHNs with charge -12 , an extra scalar with charge 2 that gives mass to the Majorana pair $(1,-3)$ and the Dirac pair $(-17,19)$ as two independent DM candidates.

All other solutions presented in Tables I and II have either one or two massless chiral fermions. They can be either extra relativistic degrees of freedom or additional DM candidates if they acquire mass from another mechanism.

Finally, the one-loop topologies must be realizations of the effective operators of D-5 or D-6 in Eq. (9), with a sufficiently rich $h_{\nu}^{\alpha i}$ structure to explain the full neutrino oscillation data. That can be guaranteed by having a rank 2 Dirac neutrino mass matrix via the inclusion of a proper set of inert scalars for each solution. For example, for the first solution in Table I corresponding to the charges $(1,-2,-3$, $5,5,-6)$, three possibilities for the charges of the scalar fields participating in the Dirac neutrino mass loop can be chosen, as shown in Table III. The labels in parentheses in the first column refer to the diagrams in Fig. 3.

Before concluding, we note that the presence of $\eta_{i}{ }^{7}$ through the first vertex from left to right in the diagrams of Fig. 3, i.e.,

\footnotetext{
${ }^{7}$ This also applies to the vectorlike fermion doublet in the realizations of topologies without $\eta$ (T1-2-A-D and T1-2-A-M) in Fig. 2.
} 
TABLE II. Same as Table I but for neutrino masses generated via D-6 operators.

\begin{tabular}{|c|c|c|c|c|c|c|c|c|c|}
\hline \multirow[b]{3}{*}{$\mathrm{N}$} & \multirow[b]{3}{*}{$\ell$} & \multirow[b]{3}{*}{$\mathrm{k}$} & \multirow{2}{*}{\multicolumn{3}{|c|}{ Solutions }} & \multicolumn{4}{|c|}{$\nu_{R}$} \\
\hline & & & & & & \multicolumn{2}{|c|}{$U(1)_{D}$} & \multicolumn{2}{|c|}{$U(1)_{X}$} \\
\hline & & & Charges & GCD & Refs. & Dirac & Majorana & Dirac & Majorana \\
\hline 6 & $(-1,-2)$ & $(-1,2)$ & $(1,1,1,-4,-4,5)$ & 1 & {$[32,43]$} & & $(-4)$ & & \\
\hline 6 & $(1,-2)$ & $(-4,1)$ & $(1,-4,-4,9,9,-11)$ & 3 & & & $(-4)$ & & \\
\hline 6 & $(2,1)$ & $(-2,-1,0)$ & $(1,-4,-8,14,14,-17)$ & 1 & & (14) & & & \\
\hline 7 & $(1,-2,1)$ & $(-9,6,3)$ & $(1,1,-4,-4,7,8,-9)$ & 1 & & & $(-4)$ & & \\
\hline 7 & $(3,1)$ & $(-1,-5,7)$ & $(2,2,-4,7,-8,-8,9)$ & 1 & & (2) & $(-8)$ & & \\
\hline 7 & $(-3,-1)$ & $(-2,-3,1)$ & $(3,3,3,-5,-5,-7,8)$ & 1 & & & & $(-5)$ & \\
\hline 7 & $(-3,-4)$ & $(-5,-7,-4)$ & $(4,4,5,-7,-8,-9,11)$ & 2 & & (4) & & & \\
\hline 8 & $(1,2,-2)$ & $(-7,3,0)$ & $(1,-7,-7,17,17,19,-20,-20)$ & 6 & & $(-20)$ & & & \\
\hline 8 & $(1,2,1)$ & $(-5,-10,-11)$ & $(1,2,2,4,-5,-5,-7,8)$ & 1 & & (2) & & & \\
\hline 8 & $(3,2,-2)$ & $(-4,-3,4,5)$ & $(1,1,-4,-4,-4,12,15,-17)$ & 4 & & & $(-4)$ & & \\
\hline 8 & $(-1,2,4)$ & $(-4,2,-3,0)$ & $(4,4,7,14,-16,-16,-22,25)$ & 4 & & (4) & $(-16)$ & & \\
\hline 8 & $(-10,-5,-15)$ & $(-10,-12,12)$ & $(5,5,5,-17,-27,-27,28,28)$ & 100 & & & & $(-27,28)$ & \\
\hline 8 & $(3,1,-3)$ & $(-12,-14,-4)$ & $(1,-3,-3,5,-11,12,12,-13)$ & 1 & & (12) & $(12)$ & & \\
\hline 8 & $(-2,0,-1)$ & $(-4,-3,-2)$ & $(1,2,2,-8,-8,12,15,-16)$ & 2 & & $(2)$ & $(-8)$ & & \\
\hline 8 & $(-2,-5,-4)$ & $(-3,-5,-2,0)$ & $(2,-3,7,-8,-8,11,14,-15)$ & 2 & & $(-8)$ & $(-8)$ & & \\
\hline 8 & $(0,-9,4)$ & $(-4,-6,-7,4)$ & $(1,-2,-4,-4,-4,15,22,-24)$ & 2 & & & $(-4)$ & & \\
\hline 8 & $(-1,0,-1)$ & $(-9,1,-1)$ & $(3,3,3,-7,17,-23,-23,27)$ & 4 & & & & $(-23)$ & \\
\hline 8 & $(0,1,0)$ & $(-1,-4,3,-4)$ & $(1,-5,-11,15,-16,20,20,-24)$ & 2 & & $(20)$ & $(20)$ & & \\
\hline 9 & $(3,-4,5)$ & $(-4,-3,1,-3)$ & $(1,-3,8,8,8,-12,-12,-17,19)$ & 4 & & & & $(-12)$ & $(-12)$ \\
\hline 9 & $(-2,6,-4)$ & $(-8,-7,6,3)$ & $(3,3,3,5,-16,22,-23,-23,26)$ & 20 & & & & $(-23)$ & $(-23)$ \\
\hline 9 & $(-9,2,3)$ & $(-1,-7,6,-9)$ & $(1,-4,5,5,-9,-9,-9,10,10)$ & 3 & & & & & $(5)$ \\
\hline 9 & $(1,4,-1)$ & $(-2,-5,-4,8)$ & $(1,1,1,2,5,-6,-6,-6,8)$ & 1 & & $(-6)$ & & & \\
\hline 9 & $(-9,6,7)$ & $(-2,4,3,1)$ & $(1,1,1,4,-9,-10,-10,11,11)$ & 3 & & $(-10)$ & & $(-10,11)$ & \\
\hline 9 & $(-3,-2,-4)$ & $(-1,-9,-7,4)$ & $(3,3,3,-4,-4,8,-11,-11,13)$ & 2 & & $(-4)$ & & $(-11,-4)$ & \\
\hline 9 & $(-3,0,-1)$ & $(-4,-1,-6,-4)$ & $(2,-3,-3,-8,-9,12,12,14,-17)$ & 3 & & (12) & $(12)$ & & \\
\hline 9 & $(4,6,4)$ & $(-3,-4,-3,5)$ & $(3,4,-10,-10,-10,12,12,13,-14)$ & 2 & & $(12)$ & & (12) & \\
\hline 9 & $(2,7,-4)$ & $(-5,-6,3,-6)$ & $(1,1,1,-4,-4,-11,18,26,-28)$ & 50 & & & $(-4)$ & & \\
\hline 9 & $(-3,-6,2)$ & $(-5,1,7,-8)$ & $(3,-4,-4,-9,-13,16,16,16,-21)$ & 90 & & & $(16)$ & $(-4)$ & \\
\hline 9 & $(5,-3,7)$ & $(-1,3,2,-4)$ & $(5,7,7,-8,-15,-15,-15,17,17)$ & 22 & & & & $(7,17)$ & \\
\hline 9 & $(-6,-3,5)$ & $(-4,-2,-6,8)$ & $(4,7,-8,9,-16,-16,-16,18,18)$ & 4 & & $(18)$ & $(-16)$ & (18) & \\
\hline 9 & $(-2,1,2)$ & $(-6,-8,7,6)$ & $(4,4,4,5,-6,-6,-6,-10,11)$ & 2 & & & & $(4,-6)$ & \\
\hline 9 & $(-9,2,-3)$ & $(-2,-8,5,2)$ & $(1,-2,-2,-4,-4,-4,17,27,-29)$ & 81 & & & $(-4)$ & & \\
\hline 9 & $(-5,-4,0)$ & $(-2,-1,-4,4)$ & $(1,-4,-4,-4,12,-14,15,18,-20)$ & 2 & & & $(-4)$ & & \\
\hline 9 & $(2,4,-2)$ & $(-1,-4,9,3)$ & $(1,-4,-4,-5,7,-9,16,23,-25)$ & 2 & & $(-4)$ & $(-4)$ & & \\
\hline
\end{tabular}

TABLE III. Possible charge assignments to obtain a light Dirac neutrino mass matrix of rank 2, for the first solution of Table I, i.e., (1, $-2,-3,5,5,-6)$. In the row T1-3-E-D-(I-I) [T1-3-E-D-(II-II)] the second (first) heavy Dirac fermion, from $\left(\chi_{L 2}\right)^{\dagger} \chi_{R 2} S^{*}\left[\left(\chi_{L 1}\right)^{\dagger} \chi_{R 1} S^{*}\right]$, does not participate directly in the neutrino loop.

\begin{tabular}{lccccccrrrr}
\hline \hline Field & $\nu_{R \alpha}$ & $\chi_{R 1}$ & $\left(\chi_{L 1}\right)^{\dagger}$ & $\eta_{1}$ & $\sigma_{1}$ & $\chi_{R 2}$ & $\left(\chi_{L 2}\right)^{\dagger}$ & $\eta_{2}$ & $\sigma_{2}$ \\
\hline T1-3-E-D-(I-II) & 5 & -2 & -3 & -2 & -2 & 1 & -6 & 1 & 1 \\
T1-3-E-D-(I-I) & 5 & -2 & -3 & -2 & -2 & 1 & -6 & -2 & -2 \\
T1-3-E-D-(II-II) & 5 & -2 & -3 & 1 & 1 & 1 & -6 & 1 & 1 & -5 \\
\hline \hline
\end{tabular}

$$
\mathcal{L} \supset y_{1 j}\left(\chi_{R 1}\right)^{\dagger} \epsilon_{a b} L_{j}^{a} \eta_{1}^{b}+\text { H.c. },
$$

imply the presence of lepton flavor violating processes, as for example $l_{j} \rightarrow l_{k} \gamma$, which is induced at one-loop level and mediated by the charged scalar $\eta_{1}^{+}$. By using the current experimental constraint on $\operatorname{Br}(\mu \rightarrow e \gamma)<5.7 \times 10^{-13}$ at $90 \%$ confidence level [67], upper bounds can be established for the product of Yukawa couplings like $\left|y_{12} y_{11}^{*}\right|$ [32]. 


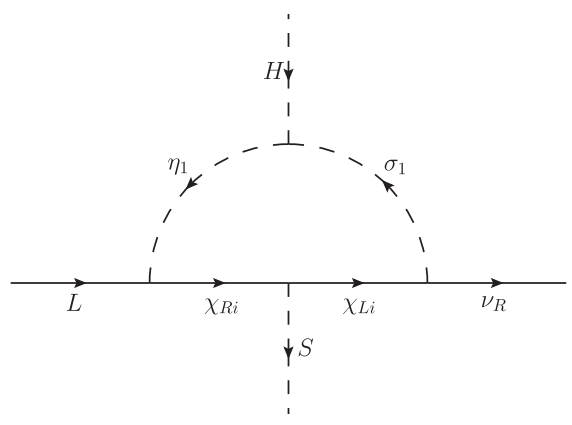

T1-3-E-D-I $(i=1)$ or T1-3-E-D-II $(i=2)$

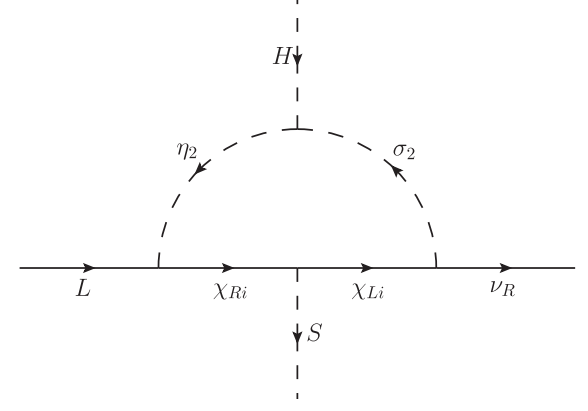

T1-3-E-D-I $(i=1)$ or T1-3-E-D-II $(i=2)$

FIG. 3. Possible contributions to the Dirac neutrino mass matrix.

\section{CONCLUSIONS}

Even if neutrino experimental data are compatible with both Majorana and Dirac neutrino masses, most of the proposals in the literature assume that neutrinos are Majorana. Having a Dirac neutrino requires the addition of singlet RHNs trivially charged under the SM gauge group and an extra symmetry spontaneously broken by a new Higgs field. Additionally, if the symmetry forbids the tree-level contribution to neutrino masses, a Dirac-seesaw mechanism can be implemented at loop level, therefore avoiding the Yukawa coupling being too small. Such symmetry can be either a dark $U(1)_{D}$ under which the SM fields are all singlets or an active $U(1)_{X}$ if the SM transforms under its action. Finally, a nonanomalous theory requires the introduction of a set of singlet fermions, with well-defined charges under the new symmetry.

Studies on one-loop Dirac neutrino masses have typically focused on finding specific anomaly-free solutions for these two kinds of symmetries. In the present work, a complete set of relevant anomaly-free solutions to the general problem of the generation of Dirac neutrino masses at one loop with chiral singlet fermions has been presented. In particular, we restricted the analysis to solutions satisfying a set of general conditions enumerated in the text, and we focused on scotogenic solutions, i.e., the ones with a DM candidate flowing in the loop. Each of the presented solutions leads to a unique model with specific phenomenological implications. The full set of solutions is shown in Tables I and II.

We found 32 and 34 sets of charges that realize the oneloop effective Dirac neutrino mass operator for dimensions 5 and 6, respectively. Solutions corresponding to both $U(1)_{D}$ and $U(1)_{X}$ gauge symmetries and Dirac or
Majorana mediators were analyzed. We emphasize that, even if most of the solutions contain one or two massless chiral fermions, there are few solutions (six and three for dimensions 5 and 6 , respectively) with all extra fermions getting mass via the spontaneous symmetry breaking of the new Higgs field. The massless fermions can either contribute to the relativistic degrees of freedom $\Delta N_{\text {eff }}$ in the early Universe [32] or acquire masses after the introduction of an extra singlet scalar, becoming independent DM candidates [68].

Finally, we note that the methodology presented can be easily applied to find the full set of anomaly-free solutions to well-defined phenomenological problems. Additionally, some particular models presented here are the subject of a detailed phenomenological study in an ongoing study [69].

\section{ACKNOWLEDGMENTS}

N.B. received funding from Universidad Antonio Nariño Grants No. 2018204, No. 2019101, and No. 2019248, the Spanish FEDER/MCIU-AEI under Grant No. FPA2017-84543-P, and the Patrimonio Autónomo-Fondo Nacional de Financiamiento para la Ciencia, la Tecnología y la Innovación Francisco José de Caldas (MinCiencias, Colombia) Grant No. 80740-4652020. The work of D. R. is supported by Sostenibilidad UdeA, the UdeA/CODI Grant No. 2017-16286 and by COLCIENCIAS through the Grant No. 1115-7765-7253. This project has received funding/support from the European Union's Horizon 2020 Research and Innovation Programme under the Marie SkłodowskaCurie Grant Agreement No. 860881-HIDDeN. D. R thanks Óscar Zapata for very useful discussions. 
[1] P. A. Zyla et al. (Particle Data Group), Review of particle physics, Prog. Theor. Exp. Phys. (2020), $083 \mathrm{C} 01$.

[2] Y. Cai, J. Herrero-García, M. A. Schmidt, A. Vicente, and R. R. Volkas, From the trees to the forest: A review of radiative neutrino mass models, Front. Phys. 5, 63 (2017).

[3] J. Sayre, S. Wiesenfeldt, and S. Willenbrock, Sterile neutrinos and global symmetries, Phys. Rev. D 72, 015001 (2005).

[4] P. Batra, B. A. Dobrescu, and D. Spivak, Anomaly-free sets of fermions, J. Math. Phys. (N.Y.) 47, 082301 (2006).

[5] K. Nakayama, F. Takahashi, and T. T. Yanagida, Numbertheory dark matter, Phys. Lett. B 699, 360 (2011).

[6] J. Heeck and H. Zhang, Exotic charges, multicomponent dark matter and light sterile neutrinos, J. High Energy Phys. 05 (2013) 164.

[7] E. Ma and R. Srivastava, Dirac or inverse seesaw neutrino masses with $B-L$ gauge symmetry and $S_{3}$ flavor symmetry, Phys. Lett. B 741, 217 (2015).

[8] A. de Gouvêa and D. Hernández, New chiral fermions, a new gauge interaction, Dirac neutrinos, and dark matter, J. High Energy Phys. 10 (2015) 046.

[9] E. Ma, N. Pollard, R. Srivastava, and M. Zakeri, Gauge $B-L$ model with residual $Z_{3}$ symmetry, Phys. Lett. B 750, 135 (2015).

[10] C. Bonilla and J. W. F. Valle, Naturally light neutrinos in Diracon model, Phys. Lett. B 762, 162 (2016).

[11] S. C. Chuliá, E. Ma, R. Srivastava, and J. W. F. Valle, Dirac neutrinos and dark matter stability from lepton quarticity, Phys. Lett. B 767, 209 (2017).

[12] S. C. Chuliá, R. Srivastava, and J. W. F. Valle, CP violation from flavor symmetry in a lepton quarticity dark matter model, Phys. Lett. B 761, 431 (2016).

[13] C. Bonilla, E. Ma, E. Peinado, and J. W. F. Valle, Two-loop Dirac neutrino mass and WIMP dark matter, Phys. Lett. B 762, 214 (2016).

[14] E. Ma and O. Popov, Pathways to naturally small Dirac neutrino masses, Phys. Lett. B 764, 142 (2017).

[15] W. Wang and Z.-L. Han, Naturally small Dirac neutrino mass with intermediate $S U(2)_{L}$ multiplet fields, J. High Energy Phys. 04 (2017) 166.

[16] W. Wang, R. Wang, Z.-L. Han, and J.-Z. Han, The $B-L$ scotogenic models for Dirac neutrino masses, Eur. Phys. J. C 77, 889 (2017).

[17] D. Borah and B. Karmakar, $A_{4}$ flavour model for Dirac neutrinos: Type I and inverse seesaw, Phys. Lett. B 780, 461 (2018).

[18] M. Hirsch, R. Srivastava, and J. W. F. Valle, Can one ever prove that neutrinos are Dirac particles? Phys. Lett. B 781, 302 (2018).

[19] C.-Y. Yao and G.-J. Ding, Systematic study of one-loop dirac neutrino masses and viable dark matter candidates, Phys. Rev. D 96, 095004 (2017); Erratum, Phys. Rev. D 98, 039901 (2018).

[20] S. C. Chuliá, R. Srivastava, and J. W. F. Valle, Generalized bottom-tau unification, neutrino oscillations and dark matter: Predictions from a lepton quarticity flavor approach, Phys. Lett. B 773, 26 (2017).

[21] C.-Y. Yao and G.-J. Ding, Systematic analysis of Dirac neutrino masses from a dimension five operator, Phys. Rev. D 97, 095042 (2018).
[22] M. Reig, D. Restrepo, J. W. F. Valle, and Ó. Zapata, Boundstate dark matter and Dirac neutrino masses, Phys. Rev. D 97, 115032 (2018).

[23] Z.-L. Han and W. Wang, $Z^{\prime}$ portal dark matter in $B-L$ scotogenic Dirac model, Eur. Phys. J. C 78, 839 (2018).

[24] C. Bonilla, S. Centelles-Chuliá, R. Cepedello, E. Peinado, and R. Srivastava, Dark matter stability and Dirac neutrinos using only Standard Model symmetries, Phys. Rev. D 101, 033011 (2020).

[25] J. Calle, D. Restrepo, C. E. Yaguna, and Ó. Zapata, Minimal radiative Dirac neutrino mass models, Phys. Rev. D 99, 075008 (2019).

[26] D. Borah, B. Karmakar, and D. Nanda, Common origin of Dirac neutrino mass and freeze-in massive particle dark matter, J. Cosmol. Astropart. Phys. 07 (2018) 039.

[27] D. Borah and B. Karmakar, Linear seesaw for Dirac neutrinos with $A_{4}$ flavour symmetry, Phys. Lett. B 789, 59 (2019).

[28] C. D. R. Carvajal and Ó. Zapata, One-loop Dirac neutrino mass and mixed axion-WIMP dark matter, Phys. Rev. D 99, 075009 (2019).

[29] S. C. Chuliá, R. Srivastava, and J. W. F. Valle, Seesaw roadmap to neutrino mass and dark matter, Phys. Lett. B 781, 122 (2018).

[30] S. Saad, Simplest radiative dirac neutrino mass models, Nucl. Phys. B943, 114636 (2019).

[31] S. Jana, P. K. Vishnu, and S. Saad, Minimal Dirac neutrino mass models from $U(1)_{\mathrm{R}}$ gauge symmetry and left-right asymmetry at colliders, Eur. Phys. J. C 79, 916 (2019).

[32] J. Calle, D. Restrepo, and Ó. Zapata, Dirac neutrino mass generation from a Majorana messenger, Phys. Rev. D 101, 035004 (2020).

[33] S. Jana, P. K. Vishnu, and S. Saad, Minimal realizations of Dirac neutrino mass from generic one-loop and two-loop topologies at $d=5$, J. Cosmol. Astropart. Phys. 04 (2020) 018.

[34] K. Enomoto, S. Kanemura, K. Sakurai, and H. Sugiyama, New model for radiatively generated Dirac neutrino masses and lepton flavor violating decays of the Higgs boson, Phys. Rev. D 100, 015044 (2019).

[35] D. Borah, D. Nanda, and A. K. Saha, Common origin of modified chaotic inflation, nonthermal dark matter, and Dirac neutrino mass, Phys. Rev. D 101, 075006 (2020).

[36] D. Restrepo, A. Rivera, and W. Tangarife, Singlet-doublet Dirac dark matter and neutrino masses, Phys. Rev. D 100, 035029 (2019).

[37] A. Dasgupta, S. K. Kang, and O. Popov, Radiative Dirac neutrino mass, neutrinoless quadruple beta decay, and dark matter in $B-L$ extension of the standard model, Phys. Rev. D 100, 075030 (2019).

[38] E. Ma, Scotogenic cobimaximal Dirac neutrino mixing from $\Delta(27)$ and $U(1)_{\chi}$, Eur. Phys. J. C 79, 903 (2019).

[39] E. Ma, Two-loop $Z_{4}$ Dirac neutrino masses and mixing, with self-interacting dark matter, Nucl. Phys. B946, 114725 (2019).

[40] S. C. Chuliá, R. Srivastava, and A. Vicente, The inverse seesaw family: Dirac and Majorana, J. High Energy Phys. 03 (2021) 248.

[41] X. Wang, Dirac neutrino mass models with a modular $S_{4}$ symmetry, Nucl. Phys. B962, 115247 (2021). 
[42] C.-F. Wong, Anomaly-free chiral $U(1)_{D}$ and its scotogenic implication, Phys. Dark Universe 32, 100818 (2021).

[43] E. Ma, Linkage of Dirac neutrinos to dark U(1) gauge symmetry, Phys. Lett. B 817, 136290 (2021).

[44] S. C. Chuliá, R. Cepedello, E. Peinado, and R. Srivastava, Scotogenic dark symmetry as a residual subgroup of standard model symmetries, Chin. Phys. C 44, 083110 (2020).

[45] P.-H. Gu and U. Sarkar, Radiative neutrino mass, dark matter and leptogenesis, Phys. Rev. D 77, 105031 (2008).

[46] B. Batell, Dark discrete gauge symmetries, Phys. Rev. D 83, 035006 (2011).

[47] Y. Farzan and E. Ma, Dirac neutrino mass generation from dark matter, Phys. Rev. D 86, 033007 (2012).

[48] C. Bonilla, E. Peinado, and R. Srivastava, The role of residual symmetries in dark matter stability and the neutrino nature, Lett. High Energy Phys. 2, 124 (2019).

[49] P. Escribano, M. Reig, and A. Vicente, Generalizing the scotogenic model, J. High Energy Phys. 07 (2020) 097.

[50] T. Appelquist, B. A. Dobrescu, and A. R. Hopper, Nonexotic neutral gauge bosons, Phys. Rev. D 68, 035012 (2003).

[51] M. D. Campos, D. Cogollo, M. Lindner, T. Melo, F. S. Queiroz, and W. Rodejohann, Neutrino masses and absence of flavor changing interactions in the 2HDM from gauge principles, J. High Energy Phys. 08 (2017) 092.

[52] A. Das, N. Okada, and D. Raut, Enhanced pair production of heavy Majorana neutrinos at the LHC, Phys. Rev. D 97, 115023 (2018).

[53] B. Holdom, Two $U(1)$ 's and $\epsilon$ charge shifts, Phys. Lett. 166B, 196 (1986).

[54] C. Cheung, J. T. Ruderman, L.-T. Wang, and I. Yavin, Kinetic mixing as the origin of light dark scales, Phys. Rev. D 80, 035008 (2009).

[55] T. Gherghetta, J. Kersten, K. Olive, and M. Pospelov, Evaluating the price of tiny kinetic mixing, Phys. Rev. D 100, 095001 (2019).
[56] J. M. Berryman, A. de Gouvêa, D. Hernández, and K. J. Kelly, Imperfect mirror copies of the standard model, Phys. Rev. D 94, 035009 (2016).

[57] J. Rathsman and F. Tellander, Anomaly-free model building with algebraic geometry, Phys. Rev. D 100, 055032 (2019).

[58] D. B. Costa, B. A. Dobrescu, and P. J. Fox, General Solution to the U(1) Anomaly Equations, Phys. Rev. Lett. 123, 151601 (2019).

[59] D. B. Costa, B. A. Dobrescu, and P. J. Fox, Chiral Abelian gauge theories with few fermions, Phys. Rev. D 101, 095032 (2020).

[60] https://pypi.org/project/anomalies/.

[61] G. Cleaver, M. Cvetič, J. R. Espinosa, L. L. Everett, and P. Langacker, Intermediate scales, mu parameter, and fermion masses from string models, Phys. Rev. D 57, 2701 (1998).

[62] P.-H. Gu and H.-J. He, Neutrino mass and baryon asymmetry from Dirac seesaw, J. Cosmol. Astropart. Phys. 12 (2006) 010.

[63] Y. Farzan, S. Pascoli, and M. A. Schmidt, Recipes and ingredients for neutrino mass at loop level, J. High Energy Phys. 03 (2013) 107.

[64] A. Falkowski, C. Gross, and O. Lebedev, A second Higgs from the Higgs portal, J. High Energy Phys. 05 (2015) 057.

[65] H. Davoudiasl, R. Kitano, G. D. Kribs, and H. Murayama, Models of neutrino mass with a low cutoff scale, Phys. Rev. D 71, 113004 (2005).

[66] K. S. Babu and G. Seidl, Simple model for $(3+2)$ neutrino oscillations, Phys. Lett. B 591, 127 (2004).

[67] J. Adam et al. (MEG Collaboration), New Constraint on the Existence of the $\mu^{+} \rightarrow e^{+} \gamma$ Decay, Phys. Rev. Lett. 110, 201801 (2013).

[68] N. Bernal, D. Restrepo, C. Yaguna, and Ó. Zapata, Twocomponent dark matter and a massless neutrino in a new $B-L$ model, Phys. Rev. D 99, 015038 (2019).

[69] N. Bernal, J. Calle, and D. Restrepo (to be published). 